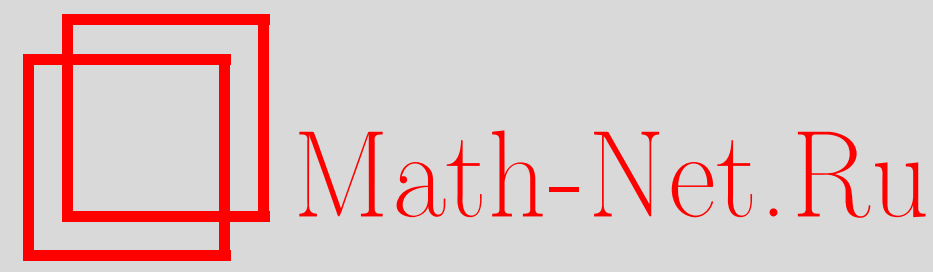

Н. Н. Ганиходжаев, Ф. М. Мухамедов, Об эргодических свойствах дискретных квадратичных стохастических процессов, определенных на алгебрах фон Неймана, Изв. РАН. Сер. матем., 2000, том 64, выпуск 5, 3-20

DOI: https://doi.org/10.4213/im302

Использование Общероссийского математического портала Math-Net.Ru подразумевает, что вы прочитали и согласны с пользовательским соглашением

http://www . mathnet.ru/rus/agreement

Параметры загрузки:

IP: 54.80 .97 .219

26 апреля 2023 г., 14:56:31 
УДК $517.98+519.21$

\author{
Н. Н. Ганиходжаев, Ф. М. Мухамедов
}

\title{
Об эргодических свойствах дискретных квадратичных стохастических процессов, определенных на алгебрах фон Неймана
}

\begin{abstract}
В настоящей работе даются необходимые и достаточные условия выполнения эргодического принципа и условия регулярности для дискретных квантовых квадратичных стохастических процессов, определенных на алгебрах фон Неймана. Устанавливается взаимосвязь таких процессов с марковскими процессами.

Библиограффия: 25 наименований.
\end{abstract}

\section{§ 1. Введение}

Изучение многих физических систем сводится к изучению марковских процессов, связанных с этими системами. Но имеются также системы, которые не описываются марковскими процессами.

Во введении опишем некоторые задачи естествознания, исследование которых приводит к квадратичным стохастическим операторам. Больцман в статье "О связи между вторым началом механической теории теплоты и теорией вероятностей в теоремах о тепловом равновесии" [1] решает следуюшую задачу, которую он формулирует так: "Из соотношений между числами различных распределений состояний рассчитать их вероятность". В качестве исследуемого тела в первой части статьи [1] берется самое простое, а именно газ, заключенный между твердыми абсолютно упругими стенками, молекулы которого представляют собой жесткие абсолютно упругие шары одинаковых радиусов и масс. Далее предполагается, что каждая молекула способна обладать определенным конечным числом скоростей, например $0,1 / q, 2 / q, \ldots, p / q$, и что в результате столкновений различные молекулы могут получить только те скорости, которые находятся среди приведенных вьше.

В [2] рассмотрена следующая обобщенная модель Больцмана. Совокупность векторов скоростей молекул как элементы $\mathbb{R}^{3}$ распределим по $n$ областям $E_{1}$, $E_{2}, \ldots, E_{n}$, образующих разбиение $\mathbb{R}^{3}$, т.е. $E_{i} \cap E_{j}=\varnothing$ при $i \neq j$ и $\bigcup_{i=1}^{n} E_{i}=\mathbb{R}^{3}$. Положим для $i=1,2, \ldots, n$

$$
x_{i}(t)=\left\{\begin{array}{c}
\text { доля молекул, скорость которых в момент } \\
\text { времени } t \text { принадлежит области } E_{i}
\end{array}\right\} \text {. }
$$

При некоторых естественных предположениях (см. [2]) можно утверждать, что

$$
\left\{\begin{array}{c}
\text { число } l-m \text { столкновений } \\
\text { за единицу времени }
\end{array}\right\}=\mu_{l m} x_{l} x_{m}
$$

(C) Н.Н. ГАНИХОДЖАев, Ф.М. Мухамедов, 2000 
для некоторого $\mu_{l m}>0$. Из числа всех $E_{l}$ молекул, т.е. молекул, скорости которых принадлежат $E_{l}$, вступающих в соударение с $E_{m}$ молекулами (число $l-m$ столкновений), обозначим через $p_{l m}^{i}$ ту часть $E_{l}$ молекул, которые рассеиваются в $E_{i}$. Очевидно, $\left(p_{l m}^{1}, p_{l m}^{2}, \ldots, p_{l m}^{n}\right)$ является вероятностным вектором для любой пары $l, m$. Тогда

$$
\left\{\begin{array}{cccc}
\text { число } & E_{l} & \text { молекул, рассеивающихся в область } E_{i} \\
\text { вследствие } & l-m \quad \text { столкновений за единицу времени }
\end{array}\right\}=p_{l m}^{i} \mu_{l m} x_{l} x_{m},
$$

откуда очевидно

$$
\begin{aligned}
& \left\{\begin{array}{c}
\text { результат изменения в популящии } E_{i} \text { молекул вследствии } \\
\text { бинарных соударений за единицу времени }
\end{array}\right\}= \\
& =\sum_{l, m=1}^{n} p_{l m}^{i} \mu_{l m} x_{l} x_{m}-\sum_{l, m=1}^{n} p_{l m}^{l} \mu_{l m} x_{l} x_{m}=\sum_{l, m=1}^{n} a_{l m}^{i} \mu_{l m} x_{l} x_{m}, \quad i=\overline{1, n},
\end{aligned}
$$

где $a_{l m}^{i}=\frac{1}{2}\left(\left(p_{l m}^{i}+p_{l m}^{l}\right)-\left(\delta_{i l}-\delta_{i m}\right)\right) \mu_{l m}$ и $\delta_{i j}-$ символ Кронекера. Таким образом,

$$
x_{i}(t+1)-x_{i}(t)=\sum_{l, m=1}^{n} a_{l m}^{i} x_{l} x_{m}
$$

и это уравнение можно свести к следующему:

$$
x_{i}(t+1)=\sum_{l, m=1}^{n} p_{l m, i} x_{l}(t) x_{m}(t)
$$

где $p_{m l, i}=a_{l m}^{i}+\delta_{m l, i}$ и

$$
\delta_{m l, i}=\left\{\begin{array}{l}
1, \quad \text { если } m=l=i, \\
\frac{1}{2}, \quad \text { если } m \neq l \text { и либо } m=i, \text { либо } l=i, \\
0 \quad \text { в остальных случаях. }
\end{array}\right.
$$

Коэффищиенты $p_{m l, i}$ удовлетворяют следующим условиям:

1) $p_{m l, i} \geqslant 0$ для всех $m, l$ и $i$;

2) $p_{m l, i}=p_{l m, i}$

3) $\sum_{i=1}^{n} p_{m l, i}=1$.

Рассмотрим некоторую биологическую популящию, т.е. замкнутое относительно размножения сообщество организмов [3]. Предположим, что каждая особь, входящая в популящию, принадлежит некоторой (единственной) из разновидностей $1,2, \ldots, n$. Шкала разновидностей такова, что разновидности родителей $i$ и $j$ однозначно определяют вероятность каждой разновидности $k$ для непосредственного потомка первого поколения. Обозначим эту вероятность (коэффищиент наследственности) через $p_{i j, k}$. Очевидно, $p_{i j, k} \geqslant 0, \sum_{k=1}^{n} p_{i j, k}=1$ для всех $i, j, k$. Предположим, что популящия настолько велика, что можно пренебречь флуктуациями частот. Тогда ее состояние можно описывать набором $x=\left(x_{1}, x_{2}, \ldots, x_{n}\right)$ вероятностей разновидностей, т.е. $x_{i}$ есть доля разновидности $i$ в популяции. При так 
называемой панмиксии, или случайном скрешивании, при фиксированном состоянии $x=\left(x_{1}, x_{2}, \ldots, x_{n}\right)$ родительские пары $i$ и $j$ образуются с вероятностью $x_{i} x_{j}$ и, следовательно,

$$
x_{k}^{\prime}=\sum_{i, j=1}^{n} p_{i j, k} x_{i} x_{j}
$$

будут полной вероятностью разновидности $k$ среди непосредственных потомков первого поколения.

Множество $S^{n-1}=\left\{x=\left(x_{1}, x_{2}, \ldots, x_{n}\right) \mid x_{j} \geqslant 0, \sum_{j=1}^{n} x_{j}=1\right\}$ называется $(n-1)$-мерным симплексом, и так как $x_{j}^{\prime} \geqslant 0$ и $\sum_{j=1}^{n} x_{j}^{\prime}=1$, то отображение $(*)$, называемое квадратичным стохастическим оператором, переводит симплекс $S^{n-1}$ в себя. Понятие квадратичного стохастического оператора впервые было сформулировано в работе [3]. В дальнейшем в работе [4] была поставлена задача изучения поведения траекторий квадратичных стохастических операторов. Невозможность создания достаточно развитых аналитических методов в силу сложных и громоздких рекурренций при изучении траекторий и необходимость проведения очень большого числа вычислений при изучении конкретных квадратичных операторов не стимулировало интерес к этой задаче.

В модели наследственной передачи, предложенной Элстоном и Стьюартом [5, c. 219], передача признака от родителей к потомству описывается тремя показателями вероятностей этой передачи:

$$
\begin{aligned}
& p_{A A, A}-\text { от родителя с генотипом } A A \text { ребенку передается аллель } A, \\
& p_{A a, A}-\text { от родителя с генотипом } A a \text { ребенку передается аллель } A, \quad(* *) \\
& p_{a a, A}-\text { от родителя с генотипом } a a \text { ребенку передается аллель } A,
\end{aligned}
$$

и тогда $p_{., a}=1-p_{., A}$.

Таким образом, исследование этой модели также сводится к изучению квадратичных стохастических операторов.

В [6]-[9] изучались предельное поведение и эргодические свойства траекторий квадратичных операторов. Квадратичные операторы, действующие на конечномерном симплексе, определяются кубическими стохастическими матрицами. В [10] В. М. Максимов исследовал кубические стохастические матрищы и описал некоторые эргодические свойства таких матриц.

В [11]-[14] определен квадратичный стохастический процесс, который естественным образом возникает при изучении некоторых моделей со взаимодействиями. Этот процесс определяется следуюшим образом. Пусть $(E, \mathscr{B})$ - измеримое пространство и $M-$ совокупность всех вероятностных мер на $(E, \mathscr{B})$. Предположим, что нам дано семейство функций $\{P(s, x, y, t, A)\}$, определенных при $t-s \geqslant 1$ для любых $x$ и $y$ из $E$ и произвольного измеримого множества $A \in \mathscr{B}$, и что семейство функций $\left\{P(s, x, y, t, A): x, y \in E, A \in \mathscr{B}, s, t \in \mathbb{R}^{+}, t-s \geqslant 1\right\}$ удовлетворяют следуюшим условиям:

1) $P(s, x, y, t, A)=P(s, y, x, t, A)$ для любых $x, y \in E$ и $A \in \mathscr{B}$;

2) $P(s, x, y, t, \cdot) \in M$ для любых фиксированных $x, y \in E$;

3) $P(s, x, y, t, A)$ как функция двух переменных $x$ и $y$ является измеримой функций по отношению $(E \times E, \mathscr{B} \otimes \mathscr{B})$ для любого фиксированного $A \in \mathscr{B}$; 
4) для начальной меры $\mu_{0} \in M$ и произвольных $s, \tau, t \in \mathbb{R}^{+}$таких, что $t-\tau \geqslant 1$ и $\tau-s \geqslant 1$, имеем:

4) А либо

$$
P(s, x, y, t, A)=\int_{E} \int_{E} P(s, x, y, \tau, d u) P(\tau, u, v, t, A) \mu_{\tau}(d v),
$$

где мера $\mu_{\tau}$ на $(E, \mathscr{B})$ определяется следующим образом:

$$
\mu_{\tau}(B)=\int_{E} \int_{E} P(0, x, y, \tau, B) \mu_{0}(d x) \mu_{0}(d y) \text { для любых } B \in \mathscr{B},
$$

4) в либо

$$
\begin{aligned}
P(s, x, y, t, A)= & \int_{E} \int_{E} \int_{E} \int_{E} P(s, x, z, \tau, d u) P(s, y, v, \tau, d w) \\
& \times P(\tau, u, w, t, A) \mu_{s}(d z) \mu_{s}(d v) .
\end{aligned}
$$

Тогда процесс, определенный функциями $P(s, x, y, t, A)$, называется квадратичным стохастическим типа (A) или типа (В) соответственно, в зависимости от того, которое из фундаментальных уравнений, 4) А или 4) В, имеет место. В этом определении функция $P(s, x, y, t, A)$ означает вероятность того, что при взаимодействии элементов $x$ и $y$ из $E$ в момент $s$ осуществится в момент $t$ один из элементов множества $A \in \mathscr{B}$. Так как для физических, химических, биологических явлений необходимо некоторое время для реализации взаимодействия, то наибольшее из таких времен примем равным 1 (см. модель Больцмана [2] или биологические модели [15]). Таким образом, вероятность $P(s, x, y, t, A)$ определена при $t-s \geqslant 1$.

Заметим, что такие процессы (т.е. квадратичные стохастические процессы (к.с.п.)) описывают физические системы, определенные выше. Но они не охватывают случаев квантовых систем, так что естественна задача определения квантовых квадратичных процессов. Отметим, что такие системы также возникают при изучении биологических и химических процессов на квантовом уровне [16], [17].

В настояшей работе мы изучаем квантовые квадратичные стохастические процессы (к.к.с.п.). В [17] рассматривались некоторые эргодические свойства таких процессов. В этой статье даются необходимые и достаточные условия справедливости эргодического принципа. Физически это означает, что описываемая этим процессом система для достаточно больших промежутков времени не зависит от начального состояния системы. Устанавливаются условия, при выполнении которых такие процессы регулярны. Выясняется как соотносятся к.к.с.п. и некоммутативные марковские процессы.

Часть результатов настоящей работы были анонсированы в работах [16], [18], [19].

\section{§ 2. Основные результаты}

1. Предварительные сведения и определения. Пусть $B(H)$ является алгеброй всех линейных ограниченных операторов на комплексном гильбертовом пространстве $H$. Слабо замкнутая *-подалгебра $\mathscr{M}$ в $B(H)$ называется алгеброй 
фон Неймана, если она содержит единичный оператор 1. Элемент $x \in \mathscr{M}$ называется положительным, если существует элемент $y \in \mathscr{M}$ такой, что $x=y^{*} y$. Совокупность всех положительных элементов $\mathscr{M}$ обозначается как $\mathscr{M}_{+}$. Линейньй функционал $\omega$ на $\mathscr{M}$ положителен, если $\omega(x) \geqslant 0$ для всех $x \in \mathscr{M}_{+}$. Положительньй функционал $\omega$ назьвается состоянием, если $\omega(\mathbf{1})=1$. Состояние $\omega$ нормально, если оно удовлетворяет равенству $\omega\left(\sup _{\alpha} x_{\alpha}\right)=\sup _{\alpha} \omega\left(x_{\alpha}\right)$ для каждой равномерно ограниченной возрастающей сети $\left\{x_{\alpha}\right\}$ положительных элементов из $\mathscr{M}$.

Пусть $\mathscr{M}$ - алгебра фон Неймана, действуюшая на гильбертовом пространстве $H$. Тогда

$$
\mathscr{M} \odot \mathscr{M}=\left\{\sum_{i=1}^{n} x_{i} \otimes y_{i} \mid x_{i}, y_{i} \in \mathscr{M}, i=\overline{1, n}, n \in \mathbb{N}\right\}
$$

является *-подалгеброй во множестве всех операторов на $H \otimes H$. Слабое (операторное) замыкание $\mathscr{M} \otimes \mathscr{M}$ на $B(H \otimes H)$ обозначается через $\mathscr{M} \otimes \mathscr{M}$ и называется тензорным произведением алгебры $\mathscr{M}$ на себя. Более подробную информацию об алгебрах фон Неймана можно найти, например, в [20].

Пусть $\mathscr{M}$ - алгебра фон Неймана. Через $S$ и $S^{2}$ обозначим совокупность всех нормальных состояний на $\mathscr{M}$ и $\mathscr{M} \otimes \mathscr{M}$ соответственно. Положим $\mathbb{N}_{0}=\mathbb{N} \cup\{0\}$. Пусть $\left\{P^{k, n}: \mathscr{M} \rightarrow \mathscr{M} \otimes \mathscr{M}, k \in \mathbb{N}_{0}, n \in \mathbb{N}, n>k\right\}$ - семейство линейных операторов и $U: \mathscr{M} \otimes \mathscr{M} \rightarrow \mathscr{M} \otimes \mathscr{M}$ - линейный оператор, определенный следуюшим образом: $U(x \otimes y)=y \otimes x$ для всех $x, y \in \mathscr{M}$.

ОПРЕДЕЛЕНИЕ 1. Будем говорить, что семейство линейных операторов $\left\{P^{k, n}\right\}$ определяет дискретный квантовый квадратичный стохастический прочесс (д.ж.ж.c.n.), если каждый оператор $P^{k, n}$ ультраслабо непрерывен и при этом выполняются следуюшие соотношения:

(i) $P^{k, n} \mathbf{1}_{\mathscr{M}}=\mathbf{1}_{\mathscr{M} \otimes \mathscr{M}}$, где $\mathbf{1}_{\mathscr{M}}$ и $\mathbf{1}_{\mathscr{M} \otimes \mathscr{M}}$ - единицы алгебр $\mathscr{M}$ и $\mathscr{M} \otimes \mathscr{M}$ соответственно;

(ii) $P^{k, n}\left(\mathscr{M}_{+}\right) \subset(\mathscr{M} \otimes \mathscr{M})_{+}$;

(iii) $P^{k, n}(\mathscr{M})$ симметрично, т.е. $P^{k, n}(\mathscr{M}) \subset\{x \in \mathscr{M} \otimes \mathscr{M}: U x=x\}$;

(iv) аналог уравнения Колмогорова-Чепмена: для начального состояния $\omega_{0} \in$ $S$ и произвольных $k \in \mathbb{N}_{0}, m, n \in \mathbb{N}$ таких, что $k<m<n$, имеем:

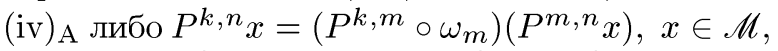

(iv) В либо $P^{k, n} x=\omega_{k} \otimes \omega_{k}\left(P^{k, m} \circ P^{k, m}\right)\left(P^{m, n} x\right), x \in \mathscr{M}$, где $\omega_{m}(x)=\omega_{0} \otimes \omega_{0}\left(P^{0, m} x\right), x \in \mathscr{M}$, и $\left(P^{k, n} \circ \omega_{n}\right)(x \otimes y):=\omega_{n}(y) P^{k, n} x$,

$$
\begin{gathered}
\omega_{n} \otimes \omega_{n}(x \otimes y \circ a \otimes b):=\omega_{n}(y) \omega_{n}(b) x \otimes a, \\
\left(P^{k, n} \circ P^{k, n}\right)(x \otimes y):=P^{k, n} x \circ P^{k, n} y .
\end{gathered}
$$

Будем говорить, что д.к.к.с.п. имеет тип (А) (соответственно тип (В)), если д.к.к.с.п. удовлетворяет равенству (iv) А (соответственно (iv)в).

При помоши так определенного д.к.к.с.п. можно задать закон взаимодействия состояний. Для $\varphi, \psi \in S$ положим

$$
V^{k, n}(\varphi, \psi)(x)=\varphi \otimes \psi\left(P^{k, n} x\right), \quad x \in \mathscr{M} .
$$


Это равенство задает правило, как при взаимодействии состояний $\varphi$ и $\psi$ в момент времени $k$ появится в момент $n$ состояние $V^{k, n}(\varphi, \psi)$. Физически взаимодействие состояний можно пояснить следующим образом. Возьмем два экземпляра физической системы, отгороженных перегородкой, где одна из них находится в состоянии $\varphi$, а другая в состоянии $\psi$. После того как перегородка убирается, новая физическая система будет в состоянии $\varphi \otimes \psi$, и после действия оператора $P^{k, n}$ образуется состояние, которое и является взаимодействием состояний $\varphi$ и $\psi$.

ЗАмечАниЕ. Если алгебра $\mathscr{M}$ абелева, т.е. $\mathscr{M}=L^{\infty}(X, \mathscr{B})$, то д.к.к.с.п. совпадает с к.с.п. Действительно, положим

$$
P(s, x, y, t, A)=\left(P^{s, t} \chi_{A}\right)(x, y), \quad x, y \in X,
$$

где $\chi_{A}$ - индикатор множества $A \in \mathscr{B}$. Тогда в силу определения 1 семейство функций $P(s, x, y, t, A)$ образует квадратичньй стохастический процесс.

Обратно, если мы имеем дискретный к.с.п. $\{P(s, x, y, t, A)\}$ на измеримом пространстве $(E, \mathscr{F})$, то можно определить д.к.к.с.п. на $L^{\infty}(E, \mathscr{F})$ следуюшим обра3ом:

$$
\left(P^{s, t} f\right)(x, y)=\int_{E} f(u) P(s, x, y, t, d u), \quad x, y \in E, \quad f \in L^{\infty}(E, \mathscr{F}) .
$$

В качестве начального состояния берется следующее состояние:

$$
\omega_{0}(f)=\int_{E} f(u) \mu_{0}(d u)
$$

где $\mu_{0}-$ начальная мера для к.с.п.

Проверим условия определения 1. Предположим, что к.с.п. типа (А). Условия (i)-(iii) очевидны. Для чисел $k \in \mathbb{N}_{0}, m, n \in \mathbb{N}$ таких, что $k<m<n$, имеем

$$
\begin{aligned}
\left(P^{k, n} f\right)(x, y) & =\int_{E} \int_{E} \int_{E} f(v) P(k, x, y, m, d u) P(m, u, z, n, d v) \mu_{m}(d z) \\
& =\int_{E} \int_{E}\left(P^{m, n} f\right)(u, z) P(k, x, y, m, d u) \mu_{m}(d z) \\
& =\left(P^{k, m} \circ \omega_{m}\right)\left(P^{m, n} f\right)(x, y)
\end{aligned}
$$

где

$$
\begin{aligned}
\omega_{m}(f) & =\int_{E} f(u) \mu_{m}(d u)=\omega_{0} \otimes \omega_{0}\left(P^{0, m} f\right), \\
\left(P^{k, m} \circ \omega_{m}\right)(f \otimes g)(x, y) & =\omega_{m}(g)\left(P^{k, m} f\right)(x, y), \quad x, y \in E, \quad f, g \in L^{\infty}(E, \mathscr{F}) .
\end{aligned}
$$

Отсюда следует справедливость уравнения (iv) А. Если к.с.п. типа (B), то уравнение (iv)в проверяется аналогично.

Таким образом, д.к.к.с.п. действительно обобщает понятия дискретного к.с.п.

Приведем примеры таких процессов.

ПРИмЕР 1. Все дискретные коммутативные квадратичные процессы являются д.к.к.с.п. 
Пример 2. Пусть $\mathscr{M}$ - алгебра фон Неймана и $T: \mathscr{M} \rightarrow \mathscr{M}$ - ультраслабо непрерывное линейное отображение такое, что: (i) $T \mathbf{1}=\mathbf{1}$, (ii) $T\left(\mathscr{M}_{+}\right) \subset \mathscr{M}_{+}$. Такое отображение называется марковским оператором. Пусть состояние $\omega_{0} \in S$ инвариантно относительно оператора $T$, т.е. $\omega_{0}(T x)=\omega_{0}(x)$ для всех $x \in \mathscr{M}$. Тогда

$$
P^{k, n} x=\frac{1}{2}\left(T^{k, n} x \otimes \mathbf{1}+\mathbf{1} \otimes T^{k, n} x\right)
$$

где

$$
T^{k, n} x=\frac{1}{2^{n-k-1}}\left(T^{n-k} x+\left(2^{n-k-1}-1\right) \omega_{0}(x) \mathbf{1}\right), \quad x \in \mathscr{M},
$$

$n>k, k \in \mathbb{N}_{0}, n \in \mathbb{N}$, является д.к.к.с.п. с начальным состоянием $\omega_{0}$.

ПРимеР 3. Пусть $\left\{T^{k, n}: k<n, k \in \mathbb{N}_{0}, n \in \mathbb{N}\right\}$ - семейство марковских операторов на $\mathscr{M}$ и $\omega_{0} \in S-$ состояние. Положим

$$
P^{k, n} x=\frac{1}{2}\left(T^{k, n} x \otimes \mathbf{1}+\mathbf{1} \otimes T^{k, n} x\right), \quad x \in \mathscr{M} .
$$

Если семейство $\left\{T^{k, n}\right\}$ удовлетворяет следующему условию:

$$
T^{k, n} x=\frac{1}{2}\left(T^{k, m} T^{m, n} x+\omega_{0}\left(T^{0, m} T^{m, n} x\right) \mathbf{1}\right), \quad x \in \mathscr{M},
$$

$k<m<n, k \in \mathbb{N}_{0}, m, n \in \mathbb{N}$, и $T^{k, k+1}=T^{0,1}$ для всех $k \in \mathbb{N}$, то $\left\{P^{k, n}\right\}$ является д.к.к.с.п. типа (А) с начальным состоянием $\omega_{0}$.

Если семейство $\left\{T^{k, n}\right\}$ удовлетворяет условию

$$
T^{k, n} x=\frac{1}{2}\left(T^{k, m} T^{m, n} x+\omega_{0}\left(T^{0, k} T^{k, m} T^{m, n} x\right) \mathbf{1}\right), \quad x \in \mathscr{M},
$$

$k<m<n, k \in \mathbb{N}_{0}, m, n \in \mathbb{N}$, и $T^{k, k+1}=T^{0,1}$ для всех $k \in \mathbb{N}$, то $\left\{P^{k, n}\right\}$ является д.к.к.с.п. типа (В) с начальным состоянием $\omega_{0}$.

Будем говорить, что д.к.к.с.п. $\left\{P^{k, n}\right\}$ однороден, если $P^{k, n}$ зависит только от $n-k$ для любых $k \in \mathbb{N}_{0}$ и $n \in \mathbb{N}$ таких, что $n>k$.

Очевидно, что д.к.к.с.п. примера 2 является однородным.

2. Эргодический принцип. В этом пункте приведем необходимые и достаточные условия выполнения эргодического принципа для дискретных квантовых квадратичных стохастических процессов.

Пусть $\left\{P^{k, n}\right\}$ - д.к.к.с.п. на алгебре фон Неймана $\mathscr{M}$. Множество всех ультраслабо непрерьвных (соответственно непрерьвных) функционалов на $\mathscr{M}$ обозначается через $\mathscr{M}_{*}\left(\right.$ соответственно $\left.\mathscr{M}^{*}\right)$. Через $P_{*}^{k, n}$ обозначим оператор, действуюший из $(\mathscr{M} \otimes \mathscr{M})_{*}$ в $\mathscr{M}_{*}$, определенный следуюшим образом:

$$
P_{*}^{k, n}(\varphi)(x)=\varphi\left(P^{k, n} x\right), \quad \varphi \in(\mathscr{M} \otimes \mathscr{M})_{*}, \quad x \in \mathscr{M} .
$$


ОПРЕДЕЛЕНИЕ 2. Будем говорить, что д.к.к.с.п. $\left\{P^{k, n}\right\}$ удовлетворяет эргодическому приниипу, если для любых $\varphi, \psi \in S^{2}$ и $k \in \mathbb{N}_{0}$ имеет место

$$
\lim _{n \rightarrow \infty}\left\|P_{*}^{k, n} \varphi-P_{*}^{k, n} \psi\right\|_{1}=0
$$

где $\|\cdot\|_{1}-$ норма на $\mathscr{M}^{*}$.

Если алгебра фон Неймана $M$ абелева, то определение эргодического принципа имеет вид [12]

$$
\lim _{n \rightarrow \infty}|P(k, x, y, n, A)-P(k, u, v, n, A)|=0
$$

для любых $x, y, u, v \in E$ и $A \in \mathscr{B}$.

Отметим, что понятие эргодического принщипа было введено впервые для марковского процесса в работах Колмогорова (см., например, [21]), а для квадратичных стохастических процессов в [12].

Лемма 1. Пусть $\left\{P^{k, n}\right\}-$ д.к.к.c.n. на алгебре фон Неймана $\mathscr{M}$, и пусть для любьх $\sigma, \varphi, \psi \in S$ u $k \in \mathbb{N}_{0}$ имеет место

$$
\lim _{n \rightarrow \infty}\left\|P_{*}^{k, n}(\sigma \otimes \varphi)-P_{*}^{k, n}(\sigma \otimes \psi)\right\|_{1}=0
$$

Тогда для любых $\varphi, \psi \in S^{2} u k \in \mathbb{N}_{0}$ справедливо

$$
\lim _{n \rightarrow \infty}\left\|P_{*}^{k, n} \varphi-P_{*}^{k, n} \psi\right\|_{1}=0
$$

ДокаЗАтЕльство. Пусть $\psi_{i}, \psi_{1, i}, \varphi, \nu, i=\overline{1, p}$, принадлежат $S$, и пусть $\left\{\lambda_{i}\right\}-$ такие числа, что $\lambda_{i} \geqslant 0, \sum_{i=1}^{p} \lambda_{i}=1$. Тогда справедливо следующее тождество:

$$
\sum_{i=1}^{p} \lambda_{i} \psi_{i} \otimes \psi_{1, i}-\varphi \otimes \nu=\sum_{i=1}^{p} \lambda_{i} \psi_{i} \otimes\left(\psi_{1, i}-\nu\right)+\left(\sum_{i=1}^{p} \lambda_{i} \psi_{i}-\varphi\right) \otimes \nu
$$

Обозначая $\psi=\sum_{i=1}^{p} \lambda_{i} \psi_{i}$, получим

$$
\sum_{i=1}^{p} \lambda_{i} \psi_{i} \otimes \psi_{1, i}-\varphi \otimes \nu=\sum_{i=1}^{p} \lambda_{i} \psi_{i} \otimes\left(\psi_{1, i}-\nu\right)+(\psi-\varphi) \otimes \nu .
$$

Следовательно,

$$
\begin{aligned}
\left\|P_{*}^{k, n}\left(\sum_{i=1}^{p} \lambda_{i} \psi_{i} \otimes \psi_{1, i}\right)-P_{*}^{k, n}(\varphi \otimes \nu)\right\|_{1} \\
\leqslant \sum_{i=1}^{p} \lambda_{i}\left\|P_{*}^{k, n}\left(\psi_{i} \otimes \psi_{1, i}\right)-P_{*}^{k, n}\left(\psi_{i} \otimes \nu\right)\right\|_{1} \\
\quad+\left\|P_{*}^{k, n}(\psi \otimes \nu)-P_{*}^{k, n}(\varphi \otimes \nu)\right\|_{1} .
\end{aligned}
$$

В силу условия леммы и условия (iii) определения 1 из (1) вытекает

$$
\left\|P_{*}^{k, n}\left(\sum_{i=1}^{p} \lambda_{i} \psi_{i} \otimes \psi_{1, i}\right)-P_{*}^{k, n}(\varphi \otimes \nu)\right\|_{1} \rightarrow 0 \text { при } n \rightarrow \infty .
$$


Таким образом, для произвольных состояний $\varphi_{j}, \varphi_{1, j}, \psi_{i}, \psi_{1, i}, \varphi, \psi \in S(i=\overline{1, p}$, $j=\overline{1, q}$ ) и чисел $\lambda_{i}, \mu_{j}$ таких, что $\sum_{i=1}^{p} \lambda_{i}=\sum_{j=1}^{q} \mu_{j}=1, \lambda_{i}, \mu_{j} \geqslant 0$, имеем

$$
\begin{aligned}
& \left\|P_{*}^{k, n}\left(\sum_{i=1}^{p} \lambda_{i} \psi_{i} \otimes \psi_{1, i}\right)-P_{*}^{k, n}\left(\sum_{j=1}^{q} \mu_{j} \varphi_{j} \otimes \varphi_{1, j}\right)\right\|_{1} \\
& \leqslant\left\|P_{*}^{k, n}\left(\sum_{i=1}^{p} \lambda_{i} \psi_{i} \otimes \psi_{1, i}\right)-P_{*}^{k, n}(\varphi \otimes \psi)\right\|_{1} \\
& \quad+\left\|P_{*}^{k, n}\left(\sum_{i=1}^{q} \mu_{j} \varphi_{j} \otimes \varphi_{1, j}\right)-P_{*}^{k, n}(\varphi \otimes \psi)\right\|_{1} \rightarrow 0 \text { при } n \rightarrow \infty .
\end{aligned}
$$

Обозначим через $G$ выпуклую оболочку множества $\{\varphi \otimes \psi: \varphi, \psi \in S\}$. Поскольку множество $G$ плотно в $S^{2}$ в $\sigma\left((\mathscr{M} \otimes \mathscr{M})_{*}, \mathscr{M} \otimes \mathscr{M}\right)$-топологии, то для произвольного $\varepsilon>0$ и для любых $\varphi, \psi \in S^{2}$ найдутся состояния $\mu, \nu \in G$ такие, что

$$
\|\varphi-\mu\|_{1}<\frac{\varepsilon}{3}, \quad\|\psi-\nu\|_{1}<\frac{\varepsilon}{3}
$$

Для состояний $\mu$ и $\nu$ существует число $n_{0}=n_{0}(k, \mu, \nu)$ такое, что

$$
\left\|P_{*}^{k, n} \mu-P_{*}^{k, n} \nu\right\|_{1}<\frac{\varepsilon}{3} \text { для всех } n \geqslant n_{0} .
$$

В силу этих неравенств и неравенства

$$
\left\|P_{*}^{k, n} \mu\right\|_{1} \leqslant\|\mu\|_{1} \quad \text { для всех } \quad \mu \in(\mathscr{M} \otimes \mathscr{M})_{*}
$$

имеем

$$
\begin{aligned}
& \left\|P_{*}^{k, n} \varphi-P_{*}^{k, n} \psi\right\|_{1} \leqslant\left\|P_{*}^{k, n} \varphi-P_{*}^{k, n} \mu\right\|_{1}+\left\|P_{*}^{k, n} \psi-P_{*}^{k, n} \nu\right\|_{1} \\
& \quad+\left\|P_{*}^{k, n} \mu-P_{*}^{k, n} \nu\right\|_{1} \leqslant\|\varphi-\mu\|_{1}+\|\psi-\nu\|_{1}+\frac{\varepsilon}{3}<\varepsilon \text { при всех } n \geqslant n_{0},
\end{aligned}
$$

откуда и следует утверждение леммы.

Будем говорить, что д.к.к.с.п. $\left\{P^{k, n}\right\}$ удовлетворяет условию $\left(\mathrm{A}_{1}\right)$ (соответственно условию $\left(\mathrm{A}_{1}\right)$ равномерно) на подмножестве $\mathscr{N} \subset S^{2}$, если существует число $\lambda \in[0,1)$ такое, что для каждой пары $\varphi, \psi \in \mathscr{N}$ и $k \in \mathbb{N}_{0}$ выполнено неравенство

$$
\left\|P_{*}^{k, n} \varphi-P_{*}^{k, n} \psi\right\|_{1} \leqslant \lambda\|\varphi-\psi\|_{1} \quad \text { для всех } \varphi, \psi \in \mathscr{N}
$$

и хотя бы для одного $n=n(k, \varphi, \psi)$.

Теорема 1. Пусть $\left\{P^{k, n}\right\}-$ д.к.к.c.n. на алгебре фон Неймана М. Следующие условия әквивалентны:

(i) $\left\{P^{k, n}\right\}$ удовлетворяет әргодическому принципу;

(ii) $\left\{P^{k, n}\right\}$ удовлетворяет условию $\left(\mathrm{A}_{1}\right)$ на $S^{2}$;

(iii) $\left\{P^{k, n}\right\}$ удовлетворяет условию $\left(\mathrm{A}_{1}\right)$ на всюду плотном подмножестве $\mathscr{F}$ множества $S^{2}$. 
ДоКАЗАТЕЛЬСтво. Импликации (i) $\Rightarrow$ (ii) $\Rightarrow$ (iii) очевидны.

Покажем, что (iii) $\Rightarrow$ (i). Рассмотрим сначала случай, когда д.к.к.с.п. является процессом типа (А).

Пусть $\left\{P^{k, n}\right\}$ является д.к.к.с.п. типа (А). Предположим, что состояния $\varphi, \psi \in$ $S^{2}$ и число $k \in \mathbb{N}_{0}$ зафиксированы. Пусть $n_{0} \in \mathbb{N}$ - произвольное число такое, что $n_{0}>k$. Обозначим

$$
\varphi_{0}=P_{*}^{k, n_{0}} \varphi \otimes \omega_{n_{0}}, \quad \psi_{0}=P_{*}^{k, n_{0}} \psi \otimes \omega_{n_{0}}
$$

Тогда ясно, что $\varphi_{0}, \psi_{0} \in S^{2}$, причем найдутся такие состояния $\mu_{0}, \nu_{0} \in \mathscr{F}$, что

$$
\left\|\varphi_{0}-\mu_{0}\right\|_{1}<\varepsilon 2^{-4}, \quad\left\|\psi_{0}-\nu_{0}\right\|_{1}<\varepsilon 2^{-4}
$$

где $\varepsilon>0$ - фиксированное произвольное число. Для этих состояний найдется $n_{1} \in \mathbb{N}$ такое, что

$$
\left\|P_{*}^{k, n_{0}+n_{1}} \mu_{0}-P_{*}^{k, n_{0}+n_{1}} \nu_{0}\right\|_{1} \leqslant \lambda\left\|\mu_{0}-\nu_{0}\right\|_{1} .
$$

Покажем, что

$$
\left\|P_{*}^{k, n_{0}+n_{1}} \varphi-P_{*}^{k, n_{0}+n_{1}} \psi\right\|_{1} \leqslant \varepsilon 2^{-2}+\lambda\|\varphi-\psi\|_{1} .
$$

В силу неравенства (2) имеем

$$
\begin{aligned}
& \left\|P_{*}^{k, n_{0}+n_{1}} \varphi-P_{*}^{k, n_{0}+n_{1}} \psi\right\|_{1} \\
& \quad=\left\|P_{*}^{n_{0}, n_{0}+n_{1}}\left(P_{*}^{k, n_{0}} \varphi \otimes \omega_{n_{0}}\right)-P_{*}^{n_{0}, n_{0}+n_{1}}\left(P_{*}^{k, n_{0}} \psi \otimes \omega_{n_{0}}\right)\right\|_{1} \\
& \quad \leqslant\left\|P_{*}^{k, n_{0}} \varphi \otimes \omega_{n_{0}}-\mu_{0}\right\|_{1}+\left\|P_{*}^{k, n_{0}} \psi \otimes \omega_{n_{0}}-\nu_{0}\right\|_{1}+\lambda\left\|\mu_{0}-\nu_{0}\right\|_{1} \\
& \quad \leqslant \varepsilon 2^{-3}+\lambda\left(\left\|\mu_{0}-\varphi_{0}\right\|_{1}+\left\|\nu_{0}-\psi_{0}\right\|_{1}+\left\|\varphi_{0}-\psi_{0}\right\|_{1}\right) \\
& \quad \leqslant \varepsilon 2^{-2}+\lambda\|\varphi-\psi\|_{1} .
\end{aligned}
$$

Предположим теперь, что найдены числа $\left\{n_{i}\right\}_{i=0}^{m}$ такие, что

$$
\left\|P_{*}^{k, K_{m}} \varphi-P_{*}^{k, K_{m}} \psi\right\|_{1} \leqslant \varepsilon 2^{-2}\left(1+2^{-1}+\cdots+2^{-(m-1)}\right)+\lambda^{m}\|\varphi-\psi\|_{1},
$$

где $K_{m}=\sum_{i=0}^{m} n_{i}$. Покажем, что это неравенство справедливо для $m+1$. Положим

$$
\varphi_{m}=P_{*}^{k, K_{m}} \varphi \otimes \omega_{K_{m}}, \quad \psi_{m}=P_{*}^{k, K_{m}} \psi \otimes \omega_{K_{m}} .
$$

Тогда $\varphi_{m}, \psi_{m} \in S^{2}$ и найдутся состояния $\mu_{m}, \nu_{m} \in \mathscr{F}$ такие, что

$$
\left\|\varphi_{m}-\mu_{m}\right\|_{1}<\varepsilon 2^{-(m+4)}, \quad\left\|\psi_{m}-\nu_{m}\right\|_{1}<\varepsilon 2^{-(m+4)} .
$$

Согласно условию $\left(\mathrm{A}_{1}\right)$ найдется число $n_{m+1} \in \mathbb{N}$ такое, что

$$
\left\|P_{*}^{K_{m}, K_{m+1}} \mu_{m}-P_{*}^{K_{m}, K_{m+1}} \nu_{m}\right\|_{1} \leqslant \lambda\left\|\mu_{m}-\nu_{m}\right\|_{1} .
$$


Отсюда, применяя (2), получим

$$
\begin{aligned}
\| P_{*}^{k,} & K_{m+1}(\varphi-\psi)\left\|_{1} \leqslant\right\| P_{*}^{K_{m}, K_{m+1}}\left(P_{*}^{k, K_{m}}(\varphi-\psi) \otimes \omega_{K_{m}}\right) \|_{1} \\
& \leqslant\left\|\varphi_{m}-\mu_{m}\right\|_{1}+\left\|\psi_{m}-\nu_{m}\right\|_{1}+\lambda\left\|\mu_{m}-\nu_{m}\right\|_{1} \\
& \leqslant \varepsilon 2^{-(m+3)}+\lambda\left(\left\|\mu_{m}-\varphi_{m}\right\|_{1}+\left\|\nu_{m}-\psi_{m}\right\|_{1}+\left\|\varphi_{m}-\psi_{m}\right\|_{1}\right) \\
& \leqslant \varepsilon 2^{-(m+2)}+\lambda\left(\varepsilon 2^{-2}\left(1+2^{-1}+\cdots+2^{-(m-1)}\right)+\lambda^{m}\|\varphi-\psi\|_{1}\right) \\
& \leqslant \varepsilon 2^{-2}\left(1+2^{-1}+\cdots+2^{-m}\right)+\lambda^{m+1}\|\varphi-\psi\|_{1} .
\end{aligned}
$$

Таким образом, мы доказали справедливость (3) для $m+1$, откуда в силу математической индукции следует справедливость (3) при любых $m \in \mathbb{N}$.

Теперь выберем такое $m$, что $\lambda^{m}\|\varphi-\psi\|_{1}<\varepsilon / 2$. Тогда для $n \geqslant K_{m}$ имеем

$$
n=K_{m}+r, \quad 0 \leqslant r<n_{m+1},
$$

откуда

$$
\begin{aligned}
\left\|P_{*}^{k, n} \varphi-P_{*}^{k, n} \psi\right\|_{1} & =\left\|P_{*}^{K_{m}, n}\left(P_{*}^{k, K_{m}}(\varphi-\psi) \otimes \omega_{K_{m}}\right)\right\|_{1} \leqslant\left\|P_{*}^{k, K_{m}}(\varphi-\psi)\right\|_{1} \\
& \leqslant \varepsilon 2^{-2}\left(1+2^{-1}+\cdots+2^{-(m-1)}\right)+\lambda^{m}\|\varphi-\psi\|_{1}<\varepsilon .
\end{aligned}
$$

Таким образом, утверждение теоремы доказано для д.к.к.с.п. типа (А).

Пусть теперь д.к.к.с.п. $\left\{P^{k, m}\right\}$ имеет тип (В). Пусть состояния $\varphi, \psi, \sigma \in S$ и число $k \in \mathbb{N}_{0}$ зафиксированы. Введем обозначения

$$
\begin{aligned}
& h^{\varphi}=\sigma \otimes \varphi, \quad h^{\psi}=\sigma \otimes \psi, \quad f^{\varphi}=\omega_{k} \otimes \varphi, \\
& f^{\psi}=\omega_{k} \otimes \psi, \quad g=\sigma \otimes \omega_{k}, \quad w=\omega_{k} \otimes \omega_{k}
\end{aligned}
$$

и положим для $n_{0}>k, n_{0} \in \mathbb{N}$,

$$
\varphi_{0}=P_{*}^{k, n_{0}} w \otimes P_{*}^{k, n_{0}} f^{\varphi}, \quad \psi_{0}=P_{*}^{k, n_{0}} w \otimes P_{*}^{k, n_{0}} f^{\psi} .
$$

Тогда $\varphi_{0}, \psi_{0} \in S^{2}$ и для произвольного $\varepsilon>0$ в силу плотности $\mathscr{F}$ найдутся состояния $\mu_{0}, \nu_{0} \in \mathscr{F}$ такие, что

$$
\left\|\varphi_{0}-\mu_{0}\right\|_{1}<\varepsilon 2^{-4}, \quad\left\|\psi_{0}-\nu_{0}\right\|_{1}<\varepsilon 2^{-4}
$$

Для $\mu_{0}$ и $\nu_{0}$ согласно условию $\left(\mathrm{A}_{1}\right)$ найдется число $n_{1} \in \mathbb{N}$ такое, что

$$
\left\|P_{*}^{n_{0}, n_{0}+n_{1}}\left(\mu_{0}-\nu_{0}\right)\right\|_{1} \leqslant \lambda\left\|\mu_{0}-\nu_{0}\right\|_{1} .
$$

Для любых $k \in \mathbb{N}_{0}$ и $m, n \in \mathbb{N}$ таких, что $k<m<n$, справедливо равенство

$$
P_{*}^{k, n+m}(\omega \otimes \varphi)=P_{*}^{n, n+m}\left(P_{*}^{k, n}\left(\omega_{k} \otimes \omega\right) \otimes P_{*}^{k, n}\left(\omega_{k} \otimes \varphi\right)\right), \quad \omega, \varphi \in S,
$$

которое непосредственным образом следует из уравнения (iv)в определения 1. 
Отсюда

$$
\begin{aligned}
& \left\|P_{*}^{k, n_{0}+n_{1}} f^{\varphi}-P_{*}^{k, n_{0}+n_{1}} f^{\psi}\right\|_{1} \\
& \quad=\left\|P_{*}^{n_{0}, n_{0}+n_{1}}\left(P_{*}^{k, n_{0}} w \otimes P_{*}^{k, n_{0}} f^{\varphi}-P_{*}^{k, n_{0}} w \otimes P_{*}^{k, n_{0}} f^{\psi}\right)\right\|_{1} \\
& \quad \leqslant\left\|\varphi_{0}-\mu_{0}\right\|_{1}+\left\|\psi_{0}-\nu_{0}\right\|_{1}+\left\|P_{*}^{n_{0}, n_{0}+n_{1}}\left(\mu_{0}-\nu_{0}\right)\right\|_{1} \\
& \quad \leqslant \varepsilon 2^{-2}+\lambda\|\varphi-\psi\|_{1} .
\end{aligned}
$$

Повторяя рассуждения случая (А), можно показать, что существуют числа $\left\{n_{i}\right\}_{i=1}^{m}$ такие, что

$$
\left\|P_{*}^{k, K_{m}} f^{\varphi}-P_{*}^{k, K_{m}} f^{\psi}\right\|_{1} \leqslant \varepsilon 2^{-2}\left(1+2^{-1}+\cdots+2^{-(m-1)}\right)+\lambda^{m}\|\varphi-\psi\|_{1} .
$$

Выберем $m$ так, чтобы $\lambda^{m}\|\varphi-\psi\|_{1}<\varepsilon / 2$. Для $n \geqslant K_{m}$ имеет место разложение (4). Следовательно, пользуясь (6) и (7), находим

$$
\begin{aligned}
\left\|P_{*}^{k, n} h^{\varphi}-P_{*}^{k, n} h^{\psi}\right\|_{1} & =\left\|P_{*}^{K_{m}, n}\left(P_{*}^{k, K_{m}}\left(f^{\varphi}-f^{\psi}\right) \otimes P_{*}^{k, K_{m}} g\right)\right\|_{1} \\
& \leqslant\left\|P_{*}^{k, K_{m}}\left(f^{\varphi}-f^{\psi}\right)\right\|_{1} \\
& \leqslant \varepsilon 2^{-2}\left(1+2^{-1}+\cdots+2^{-(m-1)}\right)+\lambda^{m}\|\varphi-\psi\|_{1}<\varepsilon .
\end{aligned}
$$

Таким образом,

$$
\lim _{n \rightarrow \infty}\left\|P_{*}^{k, n} h^{\varphi}-P_{*}^{k, n} h^{\psi}\right\|_{1}=0 .
$$

В силу (5) и леммы 1 получим требуемое соотношение. Теорема доказана.

3. Условие регулярности. В этом пункте изучаются вопросы регулярности д.к.к.с.п.; даются необходимые и достаточные условия регулярности однородных д.к.К.с.п.

ОПРЕДЕЛЕНИЕ 3. Говорят, что д.к.к.с.п. $\left\{P^{k, n}\right\}$ на $\mathscr{M}$ удовлетворяет условию регулярности (соответственно әкспоненциальной регулярности), если существует состояние $\mu_{1} \in S$ такое, что для любого $\varphi \in S^{2}$ и $k \in \mathbb{N}_{0}$ имеет место

$$
\lim _{n \rightarrow \infty}\left\|P_{*}^{k, n} \varphi-\mu_{1}\right\|_{1}=0
$$

(соответственно $\left\|P_{*}^{k, n} \varphi-\mu_{1}\right\|_{1} \leqslant d \exp (-b n)$ для всех $\varphi \in S^{2}$ и $n \geqslant n_{0}$ при некотором $n_{0} \in \mathbb{N}$, где $\left.d, b>0\right)$.

В случае коммутативной алгебры фон Неймана условие регулярности можно переформулировать следующим образом: существует вероятностная (т.е. нормированная) мера $\mu_{1}$ на $(E, \mathscr{B})$ такая, что для любых $x, y \in E, A \in \mathscr{B}$ и $k \in \mathbb{N}_{0}$ справедливо

$$
\lim _{n \rightarrow \infty}\left|P(k, x, y, n, A)-\mu_{1}(A)\right|=0 .
$$

Приведем пример регулярного процесса. Рассмотрим модель наследования (**) с

$$
p_{A A, A}^{(1)}=1, \quad p_{a a, a}^{(1)}=1, \quad p_{a A, a}^{(1)}=\frac{1}{2}
$$


и пусть начальное распределение является равномерным распределением, т.е. $x_{a}=$ $1 / 2, x_{A}=1 / 2$. Определим $\left\{p_{i j, k}^{(n)}\right\}, i, j, k \in\{A, a\}$, следующим образом:

$$
p_{i j, k}^{(n+1)}=\sum_{m \in\{A, a\}} \sum_{l \in\{A, a\}} p_{i j, m}^{(n)} p_{m l, k} x_{l},
$$

где $p_{i j, k}^{(1)}=p_{i j, k}, i, j, k \in\{A, a\}$. Тогда так определенный дискретный процесс является однородным к.с.п. Известно [12], что для этой последовательности существует предел, который стремится к равновесному распределению, т.е.

$$
\lim _{n \rightarrow \infty}\left|p_{i j, k}^{(n)}-\frac{1}{2}\right|=0 \text { для всех } i, j, k \in\{A, a\} .
$$

Будем говорить, что д.к.к.с.п. $\left\{P^{k, n}\right\}$ удовлетворяет условию $\left(\mathrm{A}_{2}\right)$ (соответственно равномерно) на $\mathscr{N} \in S^{2}$, если существуют число $\lambda \in(0,1]$ и состояние $\mu \in S$ такие, что для любых $\varphi \in \mathscr{N}$ и $k \in \mathbb{N}_{0}$ найдутся последовательность $\left\{\tau_{n}^{k}\right\}_{n \geqslant k+1}^{\infty} \subset \mathscr{M}_{*}^{+}$и число $n_{0} \in \mathbb{N}$, удовлетворяющие следуюшим условиям:

1) $\left\|\tau_{n}^{k}\right\|_{1} \rightarrow 0\left(\right.$ соответственно $\left.\sup _{\varphi \in \mathcal{N}}\left\|\tau_{n}^{k}\right\|_{1} \rightarrow 0\right)$ при $n \rightarrow \infty$;

2) $P_{*}^{k, n} \varphi+\tau_{n}^{k} \geqslant \lambda \mu$ для всех $n \geqslant n_{0}$.

Лемма 2. Для любых состояний $\varphi, \psi \in S^{2}$ существуют такие состояния $\zeta, \xi \in S^{2}$, что имеет место равенство

$$
\varphi-\psi=\frac{\|\varphi-\psi\|_{1}}{2}(\zeta-\xi)
$$

ДокАЗАТЕЛЬСТво. Обозначим $\gamma=\frac{\varphi-\psi}{\|\varphi-\psi\|_{1}}$. Ясно, что функционал $\gamma$ эрмитов, следовательно, существуют положительные нормальные функционалы $\gamma_{+}, \gamma_{-} \in$ $(\mathscr{M} \otimes \mathscr{M})_{*}^{+}$(см. [20, предложение 3.2.7]) такие, что $\gamma=\gamma_{+}-\gamma_{-}$. Пусть $\lambda^{+}=\left\|\gamma_{+}\right\|_{1}$, $\lambda^{-}=\left\|\gamma_{-}\right\|_{1}$. Положим $\zeta=\gamma_{+} / \lambda^{+}, \xi=\gamma_{-} / \lambda^{-}$; тогда $\gamma=\lambda^{+} \zeta-\lambda^{-} \xi$. В силу положительности $\gamma_{+}, \gamma_{-}$и равенства $\gamma(\mathbf{1})=0$ находим $\lambda^{+}=\lambda^{-}$. Из равенства

$$
\|\gamma\|_{1}=\left\|\gamma_{+}\right\|_{1}+\left\|\gamma_{-}\right\|_{1}, \quad\|\gamma\|_{1}=1
$$

вытекает, что $\lambda^{+}=\lambda^{-}=1 / 2$. Отсюда следует требуемое равенство.

ЛЕмма 3. Пусть д.к.к.c.n. $\left\{P^{k, n}\right\}$ на $\mathscr{M}$ удовлетворяет условию $\left(\mathrm{A}_{2}\right)$ на множестве R. Тогда этот д.к.к.с.п. удовлетворяет условию $\left(\mathrm{A}_{2}\right)$ на выпуклой оболочке $\mathscr{R}^{\mathrm{ch}}$ множества $\mathscr{R}$.

ДокаЗАтЕльство. Пусть $\left\{\varphi_{i}\right\}_{i=1}^{m} \subset \mathscr{R}, m \in \mathbb{N}$ и $\left\{\mu_{i}\right\}_{i=1}^{m}$ - такие числа, что $\mu_{i} \geqslant 0, \quad \sum_{i=1}^{m} \mu_{i}=1$. Для каждого состояния $\varphi_{i}(i=\overline{1, m})$ в силу условия $\left(\mathrm{A}_{2}\right)$ сушествуют последовательность функционалов $\left\{\tau_{n, i}^{k}\right\}_{n \geqslant k+1}$ и числа $n_{0, i} \in \mathbb{N}$ такие, что

$$
P_{*}^{k, n} \varphi_{i}+\tau_{n, i}^{k} \geqslant \lambda \mu \quad \forall n \geqslant n_{0, i},
$$

где $\tau_{n, i}^{k} \rightarrow 0$ при $n \rightarrow \infty$. Для состояния $\sum_{i=1}^{m} \mu_{i} \varphi_{i} \in \mathscr{R}^{\mathrm{ch}}$ положим

$$
\tau_{n}^{k}=\sum_{i=1}^{m} \mu_{i} \tau_{n, i}^{k}
$$

Ясно, что $\tau_{n}^{k} \rightarrow 0$ при $n \rightarrow \infty$ и

$$
P_{*}^{k, n}\left(\sum_{i=1}^{m} \mu_{i} \varphi_{i}\right)+\tau_{n}^{k}=\sum_{i=1}^{m} \mu_{i}\left(P_{*}^{k, n} \varphi_{i}+\tau_{n, i}^{k}\right) \geqslant \lambda \mu \quad \forall n \geqslant \max \left\{n_{0, i}: i=\overline{1, m}\right\} .
$$

Лемма доказана. 
ПРЕДЛОЖЕНИЕ 1. Пусть д.к.к.c.n. $\left\{P^{k, n}\right\}$ на алгебре фон Неймана $\mathscr{M}$ удовлетворяет условию $\left(\mathrm{A}_{2}\right)$ на $\mathscr{R}$, выпуклая оболочка которого $\sigma\left((\mathscr{M} \otimes \mathscr{M})_{*}\right.$, $\mathscr{M} \otimes \mathscr{M})$-слабо плотна в $S^{2}$. Тогда д.к.к.с.п. $\left\{P^{k, n}\right\}$ удовлетворяет әргодическому принципу.

ДокАЗАТЕльСтво. Пусть $\mathscr{R}^{\mathrm{ch}}$ - выпуклая оболочка $\mathscr{R}$. Тогда в силу леммы 3 д.к.к.с.п. $\left\{P^{k, n}\right\}$ удовлетворяет условию $\left(\mathrm{A}_{2}\right)$ на $\mathscr{R}^{\mathrm{ch}}$. Не ограничивая общности, можно считать, что $\lambda<1$. Если $\lambda=1$, то заведомо будет выполняться эргодический принщип.

Пусть $\varphi, \psi \in \mathscr{R}^{\mathrm{ch}}$ - произвольные состояния и $k \in \mathbb{N}_{0}$. В соответствии с леммой 2 для состояний $\varphi$ и $\psi$ существуют такие состояния $\zeta, \xi \in S^{2}$, что разложение (8) справедливо. Для произвольного $\varepsilon>0$ в силу плотности $\mathscr{R}^{\mathrm{ch}}$ можно найти состояния $\zeta_{1}, \xi_{1} \in \mathscr{R}^{\mathrm{ch}}$ такие, что

$$
\left\|\zeta-\zeta_{1}\right\|_{1}<\varepsilon, \quad\left\|\xi-\xi_{1}\right\|_{1}<\varepsilon .
$$

$\mathrm{B}$ силу условия $\left(\mathrm{A}_{2}\right)$ для состояний $\zeta_{1}$ и $\xi_{1}$ существует число $n_{0}=n_{0}\left(k, \zeta_{1}, \xi_{1}\right) \in \mathbb{N}$ такое, что

$$
P_{*}^{k, n} \zeta_{1}+\tau_{n}^{k} \geqslant \lambda \mu, \quad P_{*}^{k, n} \xi_{1}+\tau_{n}^{k} \geqslant \lambda \mu, \quad\left\|\tau_{n}^{k}\right\|_{1} \leqslant \frac{\lambda}{2} \quad \forall n \geqslant n_{0} .
$$

Отсюда имеем

$$
\begin{gathered}
\left\|P_{*}^{k, n} \zeta_{1}+\tau_{n}^{k}-\lambda \mu\right\|_{1}=P_{*}^{k, n} \zeta_{1}(\mathbf{1})+\tau_{n}^{k}(\mathbf{1})-\lambda \mu(\mathbf{1})=1-c_{1} \leqslant 1-\frac{\lambda}{2}, \\
\left\|P_{*}^{k, n} \xi_{1}+\tau_{n}^{k}-\lambda \mu\right\|_{1}=P_{*}^{k, n} \xi_{1}(\mathbf{1})+\tau_{n}^{k}(\mathbf{1})-\lambda \mu(\mathbf{1})=1-c_{1} \leqslant 1-\frac{\lambda}{2}
\end{gathered}
$$

при всех $n \geqslant n_{0}$. Положим

$$
\begin{aligned}
\mu_{1}^{k, n} & =\left(1-c_{1}\right)^{-1}\left(P_{*}^{k, n} \zeta_{1}+\tau_{n}^{k}-\lambda \mu\right), \\
\nu_{1}^{k, n} & =\left(1-c_{1}\right)^{-1}\left(P_{*}^{k, n} \xi_{1}+\tau_{n}^{k}-\lambda \mu\right) .
\end{aligned}
$$

Легко видеть, что $\mu_{1}^{k, n}, \nu_{1}^{k, n} \in S$ и

$$
P_{*}^{k, n} \zeta_{1}-P_{*}^{k, n} \xi_{1}=\left(1-c_{1}\right)\left(\mu_{1}^{k, n}-\nu_{1}^{k, n}\right) .
$$

Отсюда, пользуясь равенством (8), получим

$$
\begin{aligned}
& \left\|P_{*}^{k, n} \varphi-P_{*}^{k, n} \psi\right\|_{1} \\
& \quad \leqslant \frac{\|\varphi-\psi\|_{1}}{2}\left(\left\|\zeta-\zeta_{1}\right\|_{1}+\left\|\xi-\xi_{1}\right\|_{1}+\left\|P_{*}^{k, n} \zeta_{1}-P_{*}^{k, n} \xi_{1}\right\|_{1}\right) \\
& \quad \leqslant \frac{\|\varphi-\psi\|_{1}}{2}\left(2 \varepsilon+2\left(1-c_{1}\right)\right)=\left(\varepsilon+1-c_{1}\right)\|\varphi-\psi\|_{1} .
\end{aligned}
$$

В силу произвольности $\varepsilon$ имеем следующее неравенство:

$$
\left\|P_{*}^{k, n} \varphi-P_{*}^{k, n} \psi\right\|_{1} \leqslant q\|\varphi-\psi\|_{1} \quad \forall n \geqslant n_{0},
$$

где $q$ - произвольное число, удовлетворяющее условиям $1-\lambda / 2<q<1$. Таким образом, согласно теореме 1 получаем, что д.к.к.с.п. $\left\{P^{k, n}\right\}$ удовлетворяет эргодическому принципу. Предложение доказано.

Отметим, что это утверждение обобщает результаты работы [16]. 
ЛЕмма 4. Пусть $\left\{P^{k, n}\right\}$ - однородный д.к.к.c.n. на алгебре фон Неймана М. Тогда имеет место равенство

$$
\omega_{2}=\omega_{n} \quad \text { для любого } n \geqslant 2 .
$$

ДоКАЗАТЕЛЬСТво непосредственным образом следует из однородности д.к.к.с.п. $\left\{P^{k, n}\right\}$.

Из теоремы 1 и леммы 4 следует

Tеорема 2. Пусть $\left\{P^{k, n}\right\}-$ однородный д.к.к.c.n. на алгебре фон Неймана М. Тогда следующие условия эквивалентны:

(i) $\left\{P^{k, n}\right\}-$ регулярный прочесс;

(ii) $\left\{P^{k, n}\right\}$ удовлетворяет условию $\left(\mathrm{A}_{1}\right)$ на $S^{2}$;

(iii) $\left\{P^{k, n}\right\}$ удовлетворяет условию $\left(\mathrm{A}_{1}\right)$ на всюду плотном подмножестве $\mathscr{F}$ множества $S^{2}$.

Теорема 3. Пусть $\left\{P^{k, n}\right\}-$ однородный д.к.к.c.n. на алгебре фон Неймана М. Тогда следующие условия эквивалентны:

(i) $\left\{P^{k, n}\right\}$ экспоненциально регулярен;

(ii) $\left\{P^{k, n}\right\}$ удовлетворяет условию $\left(\mathrm{A}_{1}\right)$ равномерно на $S^{2}$;

(iii) $\left\{P^{k, n}\right\}$ удовлетворяет условию $\left(\mathrm{A}_{1}\right)$ равномерно на всюду плотном подмножестве $\mathscr{F}$ множества $S^{2}$.

ДокАЗАТЕЛЬСТво. Импликации (i) $\Rightarrow$ (ii) $\Rightarrow$ (iii) очевидны. Докажем импликацию (iii) $\Rightarrow$ (i). Сначала рассмотрим случай д.к.к.с.п. $\left\{P^{k, n}\right\}$ типа (А). Для произвольных $\varepsilon>0$ и $m \in \mathbb{N}$, повторяя рассуждения при доказательстве теоремы 1 и учитывая при этом однородность д.к.к.с.п., получим неравенство

$$
\left\|P_{*}^{0, m n_{0}} \varphi-P_{*}^{0, m n_{0}} \psi\right\|_{1} \leqslant \varepsilon 2^{-2}\left(1+2^{-1}+\cdots+2^{-(m-2)}\right)+\lambda^{m-1}\|\varphi-\psi\|_{1}
$$

для всех $\varphi, \psi \in S^{2}$. Следовательно, для любого $n \geqslant n_{0}$ имеем

$$
\left\|P_{*}^{0, n} \varphi-P_{*}^{0, n} \psi\right\|_{1} \leqslant \varepsilon 2^{-2}\left(1+2^{-1}+\cdots+2^{-(m-2)}\right)+\lambda^{m-1}\|\varphi-\psi\|_{1},
$$

где $m=\left[n / n_{0}\right]$. Отсюда в силу произвольности $\varepsilon$ и леммы 4 получим экспоненциальную регулярность д.к.к.с.п.

Случай д.к.к.с.п. типа (В) рассматривается аналогично. Теорема доказана.

ПреДЛОЖЕНИЕ 2. Пусть $\left\{P^{k, n}\right\}$ является регулярным (соответственно экспоненциально регулярным) д.к.к.с.п. на алгебре фон Неймана М. Тогда он удовлетворяет условию $\left(\mathrm{A}_{2}\right)$ (соответственно равномерно) на $S^{2}$.

ДокАЗАТЕльСтво. Пусть $\mu_{1} \in S-$ предельное состояние для $P_{*}^{k, n} \varphi$, где $\varphi \in$ $S^{2}$. Положим

$$
\tau_{n}^{k}=\left(P_{*}^{k, n} \varphi-\mu_{1}\right)_{-},
$$

где $P_{*}^{k, n} \varphi-\mu_{1}=\left(P_{*}^{k, n} \varphi-\mu_{1}\right)_{+}-\left(P_{*}^{k, n} \varphi-\mu_{1}\right)_{-}-$разложение Йордана (см. [20, предложение 3.2.7]). Покажем, что $\left\|\tau_{n}^{k}\right\|_{1} \rightarrow 0$ (соответственно $\sup _{\varphi \in S^{2}}\left\|\tau_{n}^{k}\right\|_{1} \rightarrow$ $0)$ при $n \rightarrow \infty$. Действительно,

$$
\left\|\tau_{n}^{k}\right\|_{1}=\left\|\left(P_{*}^{k, n} \varphi-\mu_{1}\right)_{-}\right\|_{1} \leqslant\left\|\left(P_{*}^{k, n} \varphi-\mu_{1}\right)\right\|_{1} \rightarrow 0 \text { при } n \rightarrow \infty
$$


(соответственно $\sup _{\varphi \in S^{2}}\left\|\tau_{n}^{k}\right\|_{1} \leqslant d \exp (-b n) \rightarrow 0$ при $\left.n \rightarrow \infty\right)$, поскольку $\left\{P^{k, n}\right\}$ регулярен (соответственно экспоненциально регулярен). Так как при этом

$$
P_{*}^{k, n} \varphi+\tau_{n}^{k}=\mu_{1}+\left(P_{*}^{k, n} \varphi-\mu_{1}\right)_{+} \geqslant \mu_{1} \text { при любых } n \geqslant n_{0},
$$

где $n_{0}=k+1$, отсюда следует справедливость условия $\left(\mathrm{A}_{2}\right)$ (соответственно равномерно) на $S^{2}$. Предложение доказано.

Tеорема 4. Пусть $\left\{P^{k, n}\right\}-$ однородный д.к.к.c.n. на алгебре фон Неймана М. Тогда следующие условия эквивалентны:

(i) $\left\{P^{k, n}\right\}$ регулярен (соответственно экспоненциально регулярен);

(ii) $\left\{P^{k, n}\right\}$ удовлетворяет условию $\left(\mathrm{A}_{2}\right)$ (соответственно равномерно) на $S^{2}$;

(iii) $\left\{P^{k, n}\right\}$ удовлетворяет условию $\left(\mathrm{A}_{2}\right)$ (соответственно равномерно) на подмножестве $\mathscr{R}$, выпуклая оболочка которого всюду плотна в $S^{2}$.

ДокАЗАТЕЛЬСТво. Импликация (i) $\Rightarrow$ (ii) следует из предложения 2. А импликация (ii) $\Rightarrow$ (iii) очевидна. Докажем импликацию (iii) $\Rightarrow$ (i). Из доказательства предложения 1 вытекает, что из условия $\left(\mathrm{A}_{2}\right)$ (соответственно равномерно) следует справедливость условия $\left(\mathrm{A}_{1}\right)$ (соответственно равномерно), откуда в силу теоремы 2 (соответственно теоремы 3 ) получим требуемую импликацию. Теорема доказана.

Теперь рассмотрим связь между д.к.к.с.п. и дискретным нестационарным марковским процессом. Напомним, что семейство линейных операторов $\left\{T^{k, n}: \mathscr{M} \rightarrow\right.$ $\left.\mathscr{M} \mid k \in \mathbb{N}_{0}, n \in \mathbb{N}\right\}$ называется дискретным нестационарныцм марковским прочессом, если для любых $k \in \mathbb{N}_{0}$ и $n \in \mathbb{N}$ выполнены условия:

(i) $T^{k, n} \mathbf{1}_{\mathscr{M}}=\mathbf{1}_{\mathscr{M}}$;

(ii) $T^{k, n}\left(\mathscr{M}_{+}\right) \subset \mathscr{M}_{+}$;

(iii) для любого $m \in \mathbb{N}$ такого, что $k<m<n$, справедливо уравнение Колмогорова-Чепмена

$$
T^{k, n} x=T^{k, m} T^{m, n} x, \quad x \in \mathscr{M} .
$$

ПРЕДЛОЖЕНИЕ 3. Пусть $\left\{P^{k, n}\right\}-$ д.к.к.c.n. на алгебре фон Неймана $\mathscr{M}$. Тогда семейство операторов $\left\{Q^{k, n}: \mathscr{M} \rightarrow \mathscr{M} \mid k \in \mathbb{N}_{0}, n \in \mathbb{N}\right\}$, определенное как

$$
Q^{k, n} x=\omega_{k}\left(P^{k, n} x\right), \quad x \in \mathscr{M},
$$

где $\omega_{k}(a \otimes b)=b \omega_{k}(a), \quad a, b \in \mathscr{M}$, является дискретныцм нестационарным марковским прочессом.

ДокАЗАТЕЛЬСтво. Предположим, что $\left\{P^{k, n}\right\}$ типа (А). Тогда

$$
Q^{k, n} Q^{n, m} x=\omega_{k}\left(\left(P^{k, n} \circ \omega_{n}\right)\left(P^{n, m} x\right)\right)=Q^{k, m} x, \quad x \in \mathscr{M},
$$

т.е. $Q^{k, n}$ является дискретным нестационарным марковским процессом.

Теперь предположим, что $P^{k, n}$ имеет тип (В). Тогда так как

$$
\begin{aligned}
\omega_{k} \otimes \omega_{k} \circ P^{k, n} x & =\omega_{k} \otimes \omega_{k}\left(P^{k, n} x\right) \\
& =\left(\left(\omega_{0} \otimes \omega_{0} \circ P^{0, k}\right) \otimes\left(\omega_{0} \otimes \omega_{0} \circ P^{0, k}\right)\right)\left(P^{k, n} x\right) \\
& =\omega_{0} \otimes \omega_{0}\left(\omega_{0} \otimes \omega_{0}\left(P^{0, k} \circ P^{0, k}\right)\left(P^{k, n} x\right)\right) \\
& =\omega_{0} \otimes \omega_{0}\left(P^{0, n} x\right)=\omega_{n}(x), \quad x \in \mathscr{M},
\end{aligned}
$$


TO

$$
\begin{aligned}
Q^{k, m} x & =\omega_{k}\left(P^{k, m} x\right)=\omega_{k}\left(\omega_{k} \otimes \omega_{k}\left(P^{k, n} \circ P^{k, n}\right)\left(P^{n, m} x\right)\right) \\
& =\omega_{k}\left(\left(\omega_{k} \circ P^{k, n} \otimes \omega_{k} \circ P^{k, n}\right)\left(P^{n, m} x\right)\right) \\
& =\left(\omega_{k} \circ P^{k, n} \otimes\left(\omega_{k} \otimes \omega_{k} \circ P^{k, n}\right)\right)\left(P^{n, m} x\right) \\
& =\left(\omega_{k} \circ P^{k, n} \otimes \omega_{n}\right)\left(P^{n, m} x\right) \\
& =\omega_{k} \circ P^{k, n}\left(\omega_{n} \circ P^{n, m} x\right)=Q^{k, n} Q^{n, m} x, \quad x \in \mathscr{M},
\end{aligned}
$$

где $\omega_{k} \circ P^{k, n} x=\omega_{k}\left(P^{k, n} x\right)$. Таким образом, верно равенство $Q^{k, n} Q^{n, m}=Q^{k, m}$. Предложение доказано.

В случае коммутативных квадратичных процессов аналогичный результат доказан в [22].

В заключение отметим, что в силу предложения 3 все доказанные выше эргодические теоремы справедливы и для некоммутативных нестационарных цепей Маркова, так что эти результаты по существу обобщают результаты работ [23]-[25], где аналогичные теоремы были доказаны для коммутативных стационарных цепей Маркова.

\section{Список литературы}

1. Больцман Л. Избранные труды. М.: Наука, 1984.

2. Janks R. D. Quadratic differential systems for interactive population models // J. Diff. Equation. 1985. V. 5. № 3. P. 497-514.

3. Бернштейн C.H. Решение одной математической проблемы, связанной с теорией наследственности // Уч. зап. Н.-И. каф. Укр. отд. мат. 1924. №1. С. 83-115.

4. Улам C. М. Нерешенные математические проблемы. М.: Наука, 1964.

5. Генетика и наследственность: Сб. статей. М.: Мир, 1987.

6. Kesten H. Quadratic transformations: a model for population growth. I, II // Adv. Appl. Prob. 1970. № 2. P. 1-82; 1970. № 2. P. 179-228.

7. Любич Ю. И. Основные понятия и теоремы эволюционной генетики свободных популяций // УМН. 1971. Т. 26. № 5. С. 51-116.

8. Сарымсаков Т. А., Ганиходжаев Р. Н. Эргодический принцип для квадратичных стохастических операторов // Изв. АН УзССР. Сер. физ.-мат. 1979. №6. С. 34-39.

9. Валландер C.C. О предельном поведении последовательности итераций некоторых квадратичных преобразований // ДАН СССР. 1972. Т. 202. № 3. С. 515-517.

10. Максимов B. М. Кубические стохастические матрицы и их вероятностные интерпретации // Теор. вероятн. и ее примен. 1996. Т. 41. № 1. С. 89-106.

11. Сарымсаков T.A., Ганиходжаев H. Н. Аналитические методы в теории квадратичных стохастических операторов // ДАН СССР. 1989. Т. 305. № 5. С. 1052-1056.

12. Сарымсаков Т. А., Ганиходжаев Н. Н. Об эргодическом принципе для квадратичных процессов // ДАН СССР. 1991. Т. 316. №6.

13. Sarymsakov T.A., Ganikhodzhaev N.N. Analytic methods in the theory of quadratic stochastic processes // J. Ther. Prob. 1990. V. 3. № 1. P. 51-70.

14. Ganikhodzhaev N. N. On stochastic processes generated by quadratic operators // J. Ther. Prob. 1991. V. 4. № 4. P. 639-653.

15. Любич Ю. И. Математические структуры в популяционной генетике. Киев: Наук. думка, 1983.

16. Ганиходжаев Н.Н., Мухамедов Ф. М. О квантовых квадратичных стохастических процессах // ДАН Респ. Узб. 1997. № 3. С. 13-16.

17. Ганиходжсаев Н.H., Мухамедов Ф. М. О квантовых квадратичных стохастических процессах и некоторые эргодические теоремы для таких процессов // Узб. матем. журн. 1997. № 3. C. 8-20. 


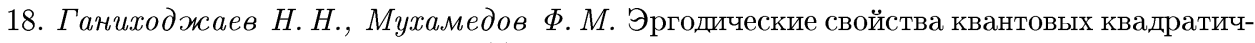
ных стохастических процессов // УМН. 1998. Т. 53. №6. С. 243-244.

19. Ганиходжаев Н.Н., Мухамедов Ф. М. Условия регулярности квантовых квадратичных стохастических процессов // Докл. РАН. 1999. Т. 365. № 3. С. 301-303.

20. Браттели У., Робинсон Д. Операторные алгебры и квантовая статистическая механика. М.: Мир, 1982.

21. Колмогоров А.Н. Об аналитических методах в теории вероятностей // УМН. 1938. № 5. C. $5-51$.

22. Ганиходжаев H. Н. Об усреднениях квадратичных стохастических процессов // ДАН У

23. Сарымсаков Т.А., Зимаков Н. П. Эргодические свойства марковских операторов на упорядоченных нормированных пространствах с базой // Операторные алгебры и функциональные пространства. Ташкент: Фан, 1988. С. 45-53.

24. Карташев Н. В. О неравенствах и теоремах эргодичности и устойчивости цепей Маркова с общим фазовым пространством. I // Теор. вероятн. и ее примен. 1985. Т. 30. № 2. C. $260-270$.

25. Аюпов Ш. А., Сарымсаков Т. А. Об однородных марковских цепях на полуполях // Теор. вероятн. и ее примен. 1981. Т. 26. № 3. С. 521-532.

Поступило в редакцию

21.VII.1999 